\title{
Management of seckel syndrome: a pediatric case report
}

\author{
Abstract \\ Sickle syndrome is a rare autosomal recessive disorder with incidence of 1:10,000 live \\ born cases. It is characterized by proportionate dwarfism, delayed mental development, \\ microcephaly and typical bird like facial appearance hence also called as Bird headed \\ dwarfism. Characteristic skeletal anomalies include premature closure of the cranial sutures \\ and fifth finger clinodactyly. Mental deficiency is present in half of the cases with levels of \\ IQ below 50. Head circumference is the most retarded parameter. The cerebellum is small \\ with a simple primitive convolutional pattern. Orodental findings include retrognathia, \\ hypodontia, enamel hypoplasia, crowding, cleft palate and Class II malocclusion. This case \\ report highlights the various clinical features and management in a pediatric patient with \\ Sickle syndrome.
}

Volume 8 Issue 5 - 2017

\author{
Mridula Goswami, Tanvi Aggarwal \\ Department of Pedodontics and Preventive Dentistry, Maulana \\ Azad Institute of Dental Sciences, India
}

Correspondence: Tanvi Aggarwal, Department of Pedodontics and Preventive Dentistry, Maulana Azad Institute of Dental Sciences, BSZ Marg, New Delhi, India, Email aggarwaltanvi89@gmail.com

Received: October 05, 2017 | Published: November 06, 2017

Keywords: proportionate dwarfism, microcephaly, bird headed dwarfism, hypoplasia

\section{Introduction}

Sickle syndrome was first defined by Helmut PG Sickle in 1960 based on 13 cases from the literature and two personally studied cases. ${ }^{1}$ It is a rare autosomal recessive condition with a reported incidence of 1:10,000 live born children. ${ }^{2}$ There are $25 \%$ chances of recurrence in subsequent siblings. ${ }^{3}$ The male to female sex ratio is 9:11 and both sexes are equally severely affected. ${ }^{4}$ Characteristic features of this syndrome include severe intrauterine and postnatal growth retardation, microcephaly, mental retardation, and typical facial appearance with beaklike protrusion of the midface (bird headed). The term "birdheaded dwarf" was introduced by Rudolf Virchow in the context of proportionate dwarfism with low birth weight, mental retardation, a pointed nose, and micrognathia. Limb anomalies, dislocation of femoral heads, scoliosis, and gastrointestinal malformation are some of the associated features. Orodental findings include retrognathia, hypodontia, enamel hypoplasia, crowding, cleft palate and Class II malocclusion. ${ }^{5}$ This case report highlights the various clinical features and management in a pediatric patient with Sickle syndrome.

\section{Case report}

A ten year old female patient reported to the department of Pedodontics and Preventive Dentistry, with a chief complaint of decayed teeth and irregularly placed upper and lower front teeth. Patient presented with good verbal behavior and was active during the visit. Birth history revealed that the patient was born at full-term caesarian delivery to non-consanguineous parents. Weight of the patient at birth was $2.5 \mathrm{~kg}$ and height was $48 \mathrm{~cm}$ with $30 \mathrm{~cm}$ head circumference. The CT examination at birth revealed early closure of sagittal suture, partial closure of coronal suture and complete closure of lambdoid and metopic suture that made a diagnosis of Primary microcephaly with secondary craniosynostosis. Past medical history revealed that there were two episodes of seizures in recent years for which she underwent treatment. Developmental history revealed delayed developmental milestones with good reading skills but poor in writing. After examination and proper treatment that included restorations and planned extractions of grossly decayed primary teeth, patient's parents were advised to seek genetic counseling before second pregnancy. Family history revealed that patient has one younger brother who is normal in growth and development.

\section{Examination}

Presently at the age of sixteen years, patient had short stature with height being $130 \mathrm{~cm}$ and weight was $26.20 \mathrm{~kg}$ with head circumference of $42 \mathrm{~cm}$. On physical examination, patient presented with receding forehead, prominent eyes, pointed triangular nose, and micrognathia, the characteristic features of "bird-headed dwarf" (Figure 1). On extraoral examination the lips were competent and micrognathia was seen. On intraoral examination, at the age of ten there were multiple carious teeth and overretained deciduous teeth resulting in crowding in both the arches. At present that is at the age of sixteen, Class II dentoskeletal pattern with deep bite and severe crowding in upper and lower arch was observed (Figures 2-4). The palate was high arched and narrow. No abnormal bony undercuts or periodontal disease was observed. Investigation of the orthopantomogram and periapical radiographs revealed impacted right maxillary canine and buccally erupting right mandibular premolar due to severe crowding. Dental treatment plan included oral prophylaxis, restoration of carious teeth, planned extraction of primary teeth which were over retained, pit and fissure sealant application, fluoride application, and orthodontic alignment of teeth. Patient was educated and motivated for brushing twice daily and healthy oral hygiene practices. Hence we could help in easy transformation from mixed to permanent dentition by regular counseling to child and parents in maintenance of orodental health. 


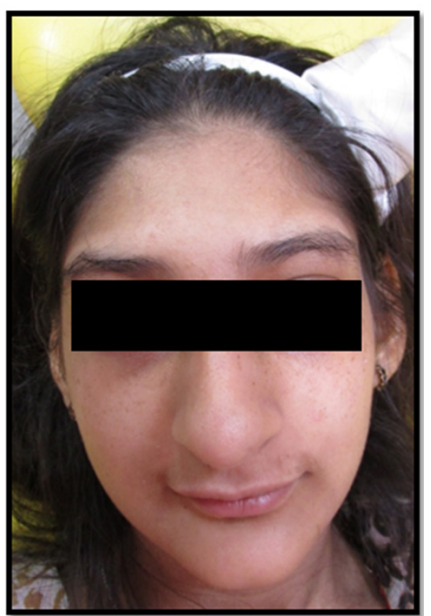

Figure I

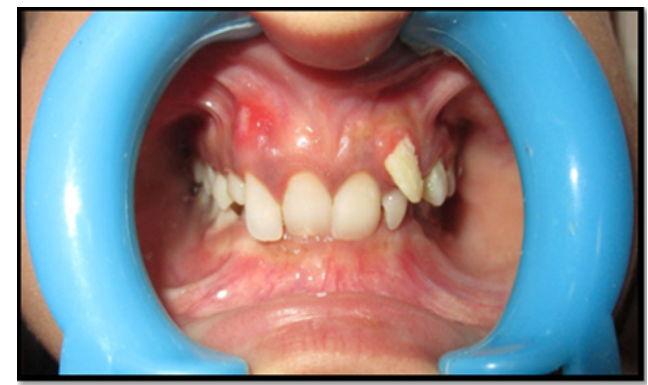

Figure 2

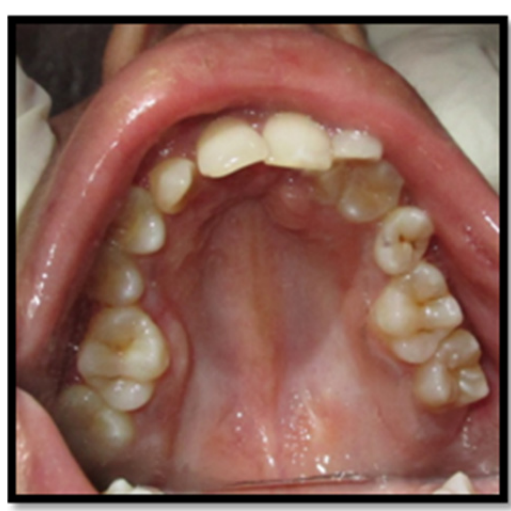

Figure 3

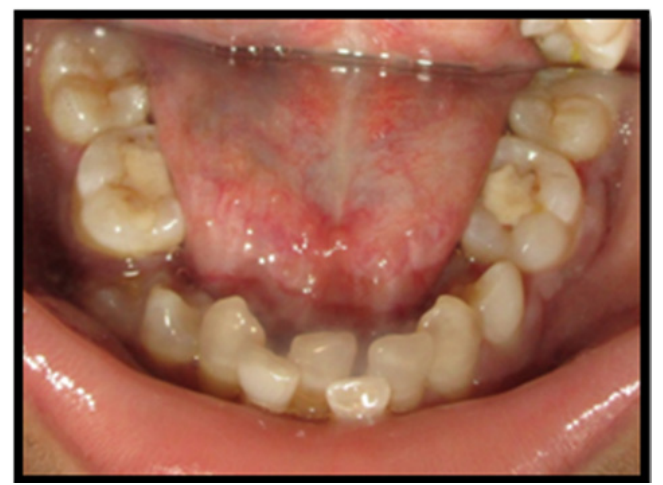

Figure 4

\section{Discussion}

Sickle syndrome is a rare constellation of malformations, presumably inherited as an autosomal recessive inherited trait. This syndrome is a heterogeneous form of primordial dwarfism. The synonyms of this syndrome include Sickle dwarfism, Bird-headed dwarfism, Nanocephalic dwarfism, and Microcephalic primordial dwarfism. ${ }^{3}$. The etiopathogenesis of this syndrome is still topic of research and linkage studies may hopefully allow the localization of the gene, which will enable the cloning and identify the underlying pathogenesis, which is responsible for the features of the Sickle syndrome. The mode of inheritance in Sickle syndrome is thought to be autosomal recessive. It can be due to increased chromosomal instability or chromosomal breakage. ${ }^{6}$ Chromosomal aberrations causing Sickle syndrome have been reported in earlier studies in genes 2q33.3-34, 18p11.31-q11.2, and 3q22.1-q24. ${ }^{6}$ In the present case, chromosomal gene mapping revealed normal karyotype. The male to female sex ratio is 9:11. Sickle syndrome encompasses a number of facial and brain abnormalities possibly associated with prenatal and postnatal growth restriction. ${ }^{7}$ The syndrome is characterized by intrauterine growth retardation (average birth weight $1.5 \mathrm{~kg}$ ) and severe proportionately short stature with severe microcephaly. In the present case, developmental history revealed delayed developmental milestones and severe growth retardation. Weight of the patient at birth was $2.5 \mathrm{~kg}$, the weight at the time of examination being $26.20 \mathrm{~kg}$ with height $130 \mathrm{~cm}$ and head circumference $42 \mathrm{~cm}$ that is very low as compared to normal girl of same age (weight $53 \mathrm{~kg}$, height- $162 \mathrm{~cm}$ ). Clinical facial features of this syndrome include "bird-header profile" with receding forehead, large eyes, beak-like protrusion of the nose, narrow face, receding lower jaw, and micrognathia. In the present case, the patient presented with characteristic features of a "bird-headed profile." Other occasional features include high-arched palate, cleft palate, malocclusion, enamel hypoplasia, premature closure of cranial sutures secondary to diminished brain growth, antimongoloid slant of palpebral fissures, dysplastic ears, clinodactyly of the fifth fingers, cryptorchidism, clitoridomegaly, hirsutism, agenesis of corpus callosum, pachygyria, retarded bone age, frequent hip dysplasia, dislocation of the head of radius, low-set ears, and eleven pairs of ribs. ${ }^{8}$ In addition, abnormalities have been found in cardiovascular, hematopoietic, and endocrine, as well as nervous system. The craniofacial features of Sickle syndrome allow its differentiation from other syndromes of growth deficiency with microcephaly, such as Dubowitz syndrome, Fetal alcohol syndrome, Trisomy 18 syndrome, DeLange syndrome, and Fanconi syndrome. Children affected with Sickle syndrome do have a normal lifespan till the age of 75years although they often have profound mental and physical deficits. ${ }^{4}$ When a first child is born with Sickle syndrome, occurrence of the disease can be suspected in second child by the observation of intrauterine growth retardation with microcephaly in the second trimester of pregnancy. Early molecular antenatal diagnosis can be performed for a couple who had a first child with Sickle syndrome if the familial mutations have been identified. ${ }^{7}$ In the present case the child has been followed for six years for physical growth, mental progress, orodental condition and behavioral aspects. Initially she was slow in physical and mental growth but over the time due to parental support and regular learning in school with special attention the condition has improved manifolds though not as per other children of her age. The dental checkup was not in the family's priority list hence regular motivation and recall visits for positive reinforcement was required that resulted in considerable improvement with age. 


\section{Conclusion}

This case shows the importance of awareness of this rare syndrome. Antenatal diagnosis with ultrasonography and karyotype analysis is of utmost importance for early diagnosis. Along with medical, dental preventive plan should be incorporated for such cases from the beginning. Preventive treatment modalities in such cases avoid unnecessary pain, general anesthesia procedure, and medical complications due to patient's limitations. Oral treatment should be provided in phases where after emergency treatment, a scheduled restorative and periodontal treatment should be done. As pediatric dentists, behavioral problems should be dealt with patience and motivation for good oral health should be regularly reinforced. These children undergo treatment on long term basis and hence become a good candidate for repetitive counseling sessions by Pedodontists. Pedodontist should target not only orodental health but should also instill a positive attitude in child for overall growth and development. We should commit ourselves in providing compassionate dental care and life long oral health counseling to patient and their parents with emphasis on dentist- patients team approach for a better life of such individuals.

\section{Acknowledgments}

None.

\section{Conflicts of interest}

The authors declares that there is no conflict of interest.

\section{Funding}

None.

\section{References}

1. Cherian MP. Seckel cell syndromes or Seckel variants? Ann Saudi Med. 2004;24(6):469-472.

2. Kutlu R, Alkan A, Kutlu O, et al. Seckel Syndrome with Polyarteritis Nodosa. Indian Pediatrics. 2004;41(17):1158-1161.

3. Garg R, Grewal A, Mittal R, et al. Palatoplasty in a patient with Seckel syndrome. Ann Maxillofac Surg. 2012;2(1):63.

4. Seymen F, Tuna B, Kayserili H. Seckel syndrome: report of a case. J Clin Pediatr Dent. 2002;26(3):305-309.

5. Parent P, Moulin S, Munck MR, et al. Bird headed dwarfism in Seckel syndrome. Nosologic difficulties. Arch Pediatr. 1996;3(1):55-62.

6. Borglum AD, Balslev T, Haagerup A, et al. A new locus for Seckel syndrome on hromosome 18p11.31-q11.2. Eur $J$ Hum Genet. 2001;9(10):753-737.

7. Napolitano R, Maroutti GM, Quarantelli M, et al. Prenatal diagnosis of Seckel Syndrome on 3-dimensional sonography and magnetic resonance imaging. J Ultrasound Med. 2009;28(3):369-374.

8. Sauk JJ, Litt R, Espiritu CE, et al. Familial bird-headed dwarfism (Seckel's syndrome). J Med Genet. 1973;10(2):196-198. 